\title{
Mismatched Filter Design using Improved Cuckoo Search Algorithm for Optimum Detection
}

\author{
K. Renu, P. Rajesh Kumar
}

\begin{abstract}
In radar signal processing pulse compression has been extensively used which solves the problem of maintaining simultaneously high transmit energy of long pulse and large range resolution of short pulse. The concept of pulse compression can be best understood from matched filtering that determines the ratio of peak of the sidelobe to peak value of mainlobe. But the resolution of weak targets from stronger one is difficult due to range sidelobes in the auto-correlation pattern of matched filter. With this idea of reducing these sidelobes, various optimization techniques are used. This paper represents a method to optimize the performance of chaotic sequence using mismatched filter. The optimization completely depends on the design of coefficients of mismatched filter at the receiver side. Here improved cuckoo search method is used instead of Lévy flight cuckoo search with the differential evolution technique to complete the design of cascaded mismatched filter. Finally, improved results are obtained as compared to Lévy flight method of cuckoo search.
\end{abstract}

Keywords: Pulse Compression, Mismatched filter (MMF), Peak Sidelobe Ratio (PSLR), Improved Cuckoo Search algorithm (ICS).

\section{INTRODUCTION}

$\mathrm{Pu}_{\mathrm{u}} \mathrm{c}$ signal and short pulses as carrier signal that results in reduced transmitted power and desired range resolution. This technique provides better accuracy and high range resolution with wide application in radar, sonar and navigation and seismic exploration systems. Ideally a radar signal should be designed to produce high resolution, high power, low probability of interference, low cost of design and simplicity of generation [1]. Now a day the miniature of sonar and radar are used in various areas such as autopilots of the cars, surveillance radars and control systems, spy vehicles and planes etc. For proper functioning of these systems the signals in these must have strong conditions particularly in their correlation functions (CF). The auto-correlation function (ACF) determines the dynamic measures like range, scale, spectrum etc. of radar and sonar images. Whereas cross-correlation function of pairs of

Revised Manuscript Received on December 13, 2019

* Correspondence Author

K. Renu", Research scholar, Department of Electronics and Communication Engineering, Andhra University, Visakhapatnam, India. Email: renuengg12@gmail.com.

P. Rajesh Kumar, Dept. of ECE, College of Engineering, AUCE, Andhra University, Visakhapatnam, India, Email: rajeshauce@gmail.com signals which are transmitted by radar, sonar should be approximately zero. Sidelobe suppression is the main aim of Matched and mismatched filters. The most interesting property of matched filter lies in producing maximum achievable SNR at the output in presence of some additive stochastic noise at the input.

At the receiver side, it combines the received echo signal with reference signal results in increasing the ratio of signal power to noise power [2]. It has been studied that the optimization of mismatched filter coefficients gives good results with various input codes such as barker code, binary codes etc. [3]- [5]. With this concept, Nunn [6] proposed that the optimization is applicable for those signal whose self $\mathrm{ACF}$ experiences minimum value of peak or integrated sidelobes. According to his ideas Levanon has recommended an optimized filter to minimize peak sidelobe ratio by using matlab function fmincon. Similarly, with longer mismatched filter minimum integrated peak sidelobe ratio was obtained using optimization techniques [7]. The length of mismatched filter is assumed to be three times longer than the input signal. Here chaotic sequences are generated and used as reference input signal. The theory of chaos is best suitable for chaotic system that highly depends on its initial conditions [8]. The study of chaos with differential evolution [9], genetic algorithm [10], firefly algorithm [11] has been discussed. The deterministic nature of chaos makes the future behaviour completely depends on initial conditions. Chaotic dynamic system provides different sequences with a tiny disturbance in initial condition. The aim of this paper is to design a mismatched filter that uses improved cuckoo search algorithm. The output performance is evaluated by cross-correlating chaotic codes with optimized coefficients of filter. The comparison is being carried out with adaptive filter that is associated after MMF to upgrade the performance of all the testing codes. In this paper the adaptive filter coefficients are updated by using least mean square [12] and binary step size least mean square method [13].

\section{IDENTIFICATION OF PROBLEM}

The technical analysis of some optimization techniques such as particle swarm and differential evolution methods and their application to various areas including mismatched filters was suggested earlier [14], [15]. In this work the same methodology is implemented to obtain the optimum weights of mismatched filter using proposed technique. 
The generation of binary sequence in pulse compression is quite easy but it appears to be difficult for longer length code to obtain optimum performance. Hence it is essential to switch over from biphase to polyphase such as ternary sequence. The ternary sequence is the combination of $-1,0$, +1 and is given as input to matched filter.

$A=\left\{c_{0}, c_{1}, c_{2}, \ldots \ldots, c_{N-1}\right\}$

The output is in the form of auto-correlation which has one mainlobe and many sidelobes. ACF is defined by (1)

$$
R_{k}=\sum_{i=0}^{N-1-k} c_{i} c_{i+k}
$$

Where ' $\mathrm{k}$ ' ranging between 0 to $\mathrm{N}-1$. The output pattern maintains symmetrical form for zero delay. The coefficients or elements of mismatched filter are represented by

$H=\left\{h_{1}, h_{2, \ldots \ldots}, h_{M}\right\}$

The weights are real valued and $M \geq N$. The value of $(M-N)$ is always even because $M$ and $N$ values are either even or odd simultaneously. The cross-correlation $C_{k}\left(H_{s} A\right)$ between $H$ and $A$ is peak at zero delay and is asymmetric around zero delay which is defined in (2).

$$
c_{k}=\sum_{i=0}^{M-1} h_{i} c_{i-k}
$$

Where ' $\mathrm{k}$ ' ranging from -(N-1) to (M-1). Assuming $a_{i}$ exist between the range 0 to $\mathrm{N}-1$. The objective function is maximize mainlobe level which is compared with absolute value of sidelobe level. Therefore, the output of mismatched filter in terms of PSLR is defined as in (3)

$P S L R=20 * \log \frac{\| \text { maximum sidelobe level } \mid}{\| \max \left(C_{K}\right) \mid}$

The impulse weights of mismatched filter are considered as the random population. with each variable vector or solution updated with the help of Cuckoo search algorithm.

\section{METHODOLOGY}

In many industrial systems, the engineers have the challenges to take decisions on how to increase the production with reduced cost. These decisions are normally based on optimization problem. Cuckoo search algorithm is one of the updated and promising metaheuristic population based optimization technique developed by Yang and Deb in the year 2009 [16]. This algorithm is influenced by the cuckoos, which are fascinating birds that produces beautiful sounds and having capability of intrusive reproduction strategy. Cuckoo search outperforms other metaheuristic algorithms in various applications. The main reason of popularity lies in their adaptability and durability as compared to other classical optimization methods. By adjusting fewer parameters, they found appropriate for many complex optimization problems [17], [18].

The synthesis of CS algorithm is discussed with the help of local exploitative and global explorative random walk which are regulated by the switching probability. The concept of Lévy cuckoo search is discussed below.

- Each cuckoo lays one egg (solution) in a randomly chosen nest.
- The cuckoo searches for best nest based on elitist selection strategy and carries high quality eggs (best solutions) for reproduction.

- The alien eggs (worse solutions) are identified from fixed number of host nests (population) with a probability $\mathrm{P}_{\mathrm{a}} \in[0,1]$. Then the host bird will either dump the egg or abandon the nest and reconstruct a completely new nest in a different location.

- Probability $\mathrm{P}_{\mathrm{a}} \in[0,1]$. Then the host bird will either dump the egg or abandon the nest and reconstruct a completely new nest in a different location.

The new solution in local search is obtained from (4)

$$
x_{i}^{t+1}=x_{i}^{t}+\alpha \otimes H\left(P_{a}-\varepsilon\right) \otimes\left(x_{j}^{t}-x_{k}^{t}\right)
$$

Here $x_{j}^{t}$ and $x_{k}^{t}$ are randomly selected solutions with random permutation. ' $H$ ' is Heaviside function and ' $E$ ' is randomly chosen from normal distribution. However, the global random walk or simply global search is obtained by using Lévy flight and can be written as in (5)

$$
x_{i}^{t+1}=x_{i}^{t}+\alpha \otimes \operatorname{Lévy}(\lambda) .
$$

Where ' $\alpha$ ' is step size parameter whose value is greater than zero. $\otimes$ represents entry-wise product.

\section{IMPROVED CUCKOO SEARCH METHODOLOGY}

Lévy flight behaviour of cuckoo search is far better than simple random search. With the necessity of optimization of complex problems, it is required to design new algorithms as per particular problem. The searching capability is improved with a new version of CS that is used to improve the coefficients of mismatched filter. But it is to be noted that no optimization method can be generalized for all optimization problems. Cuckoo search optimization has been extensively used for global optimization in many areas [19]. This paper is designed with some modification in both local and global search to achieve a better solution. The modification includes division of generation and population in both global and local search. The new version is proposed based on these modifications. The division of population and generation increases the diversification and hence improves the performance. Many benchmark functions having been tested with this version and reported earlier [20]. In division of generation, a standard equation is used for half of the generations which gives best explorative results and some computation has been done for remaining half which gives intensive exploitation. In this paper Cauchy based global search is adopted for first half of the generation. And instead of using Lévy distribution function in global search equation of basic CS algorithm, Cauchy distribution is used that examines the search space in a better way. New solutions are developed for one half of the generation by using Cauchy distribution function as in (6).

$$
x_{i}^{t+1}=x_{i}^{t}+\alpha \otimes \text { Cauchy }(\delta)\left(x_{i}^{t}-x_{j}^{t}\right)
$$

And for second half of the generation some modifications are adapted. Further, division of population into sub groups improves the exploration on whole as well as exploitation within the group. Different search equations are used in each sub group which will change the position of members of population. 
However, improved version of $C S$ is employed with dual search in both global and local phase. Here population is divided into two groups.

\section{A. Global search}

For global search, Cauchy based equation defined in (7) is used to employ search process for first half of the population. Whereas for second half, the updation of new nest/solution is done by using (8).

$$
\begin{aligned}
& x_{i}^{t+1}=x_{i}^{t}+\alpha \otimes \text { Cauchy }(\delta)\left(x_{\text {best }}-x_{i}^{t}\right) \\
& x_{i}^{t+1}=x_{i}^{t}+\alpha \otimes \text { Cauchy }(\delta)\left(x_{\text {best }}^{t}-x_{\text {new }}^{t}\right)
\end{aligned}
$$

Where $x_{\text {new }}^{t}=\frac{v_{1}+v_{2}+v_{3}}{3}$ and $v_{1}, v_{2}$ and $v_{a}$ are obtained from (9), (10) and (11)

$$
\begin{aligned}
& v_{1}=x_{i}-S_{1}\left(V_{1} x_{\text {best }}-x_{i}^{t}\right) \\
& v_{2}=x_{i}-S_{2}\left(V_{2} x_{\text {best }}-x_{i}^{t}\right) \\
& v_{a}=x_{i}-S_{a}\left(V_{a} x_{\text {best }}-x_{i}^{t}\right)
\end{aligned}
$$

The value of $S_{1}, S_{2}$ and $S_{3}$ is obtained with following expression in (12)

$$
S=2 * n * r_{1}-n
$$

and $V$ is defined in (13) as below

$$
V=2 * r_{2}
$$

The value of ' $n$ ' is varying linearly from 2 to 0 with respect to number of iterations. The variables $r_{1}$ and $r_{2}$ are in the range 0 to 1 .

\section{B. Local search}

The procedure of dividing the population is same as global search. But the new solutions are generated with (4) for half of the population and the remaining half of the population use (14) that is defined below.

$$
x_{i}^{t}=x_{i}^{t}+F_{0}\left(\left(x_{k}^{t}-x_{i}^{t}\right)+\left(x_{m}^{t}-x_{n}^{t}\right)\right)
$$

\section{SIMULATION RESULTS}

This paper presents the detailed analysis of comparison between Lévy flight and improved cuckoo search. The performance of matched and mismatched filter is compared for different lengths of input chaotic sequence. The length of the input sequence is considered as one third of the mismatched filter length. The elements of MMF are chosen from the random population of fixed size. The simulation is being carried out for fixed number of population and iterations. As per the literature survey it has been found that the value of probability switch ranges from 0 to 1 . But best results are found with value 0.5 for various problems. Similarly, the value of ' $\alpha$ ' is chosen as 0.01 . The output response of mismatched filter using improved cuckoo method for length 20 is shown in Fig.1. It is observed that the mismatched filter response is not symmetric about main lobe.

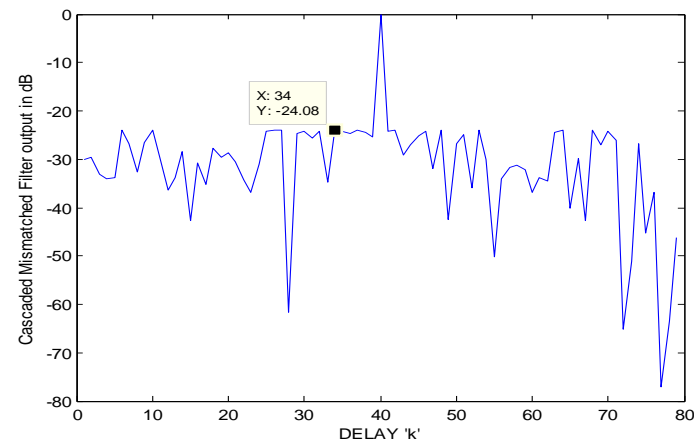

Fig.1 Cascaded Mismatched filter response of binary sequence of length 20

As the complete algorithm is based on random population, the computations on this will cause different values of PSLR values every time when the Matlab code is being executed. Apart from this the new version of CS algorithms are tested by using MATLAB in windows 8 , Intel core $\mathrm{i} 3$ processor with 4GB RAM, x64 bit personal computer. Different codes such as barker code, binary and ternary chaotic codes are used for testing. The main objective is to reduce PSLR that is defined in (3). The complete analysis for binary ternary sequence is shown in I and II. The comparison is also performed with adaptive filter connected after cascaded mismatched filter.

Table. I Improvement of PSLR of Binary Sequence by using cascaded MMF along with adaptive filter

\begin{tabular}{|c|c|c|c|c|c|l|l|}
\hline $\begin{array}{c}\text { Sequence } \\
\text { Length }\end{array}$ & $\begin{array}{c}\text { PSLR of } \\
\text { MF } \\
(\mathbf{d B})\end{array}$ & $\begin{array}{c}\text { MF } \\
\text { ASP }\end{array}$ & $\begin{array}{c}\text { PSLR of } \\
\text { DE MMF } \\
(\mathbf{d B})\end{array}$ & $\begin{array}{c}\text { PSLR of } \\
\text { Cascaded MMF } \\
\text { using DE \& } \\
\text { Lévy CS (dB) }\end{array}$ & $\begin{array}{c}\text { PSLR of } \\
\text { Cascaded MMF } \\
\text { using DE \& New } \\
\text { CS (dB) }\end{array}$ & $\begin{array}{l}\text { PSLR of } \\
\text { Adaptive } \\
\text { Filter with } \\
\text { LMS (dB) }\end{array}$ & $\begin{array}{l}\text { PSLR of } \\
\text { Adaptive } \\
\text { Filter with } \\
\text { BSSLMS (dB) }\end{array}$ \\
\hline 20 & -16.4782 & 0.15 & -22.2455 & -23.7559 & -24.0843 & -31.6297 & -37.5598 \\
\hline 25 & -18.4164 & 0.12 & -25.6436 & -28.2464 & -29.0109 & -44.5434 & -43.5884 \\
\hline 30 & -17.5012 & 0.1333 & -22.8752 & -26.4075 & -27.0840 & -29.6143 & -31.4495 \\
\hline 35 & -16.9020 & 0.1429 & -21.5543 & -25.7830 & -25.6905 & -30.2109 & -30.2219 \\
\hline 40 & -18.0618 & 0.1250 & -21.3150 & -23.8416 & -25.6901 & -29.3153 & -29.4621 \\
\hline 45 & -17.5012 & 0.1333 & -19.2216 & -21.5516 & -22.4468 & -24.6673 & -25.0377 \\
\hline 50 & -18.4164 & 0.1200 & -20.3127 & -23.3698 & -23.9746 & -25.5433 & -25.5984 \\
\hline 60 & -17.5012 & 0.1333 & -20.2337 & -22.3153 & -23.7453 & -25.8166 & -26.3209 \\
\hline 70 & -17.8171 & 0.1286 & -19.8567 & -22.1128 & -22.9599 & -24.2767 & -24.7078 \\
\hline 80 & -18.9769 & 0.1125 & -19.8137 & -22.2324 & -22.4671 & -24.9048 & -25.6998 \\
\hline 90 & -18.2570 & 0.1222 & -20.4169 & -23.1860 & -23.6681 & -25.4732 & -25.4158 \\
\hline 100 & -19.1721 & 0.1100 & -21.5543 & -25.7330 & -25.6905 & -30.2109 & -30.2219 \\
\hline
\end{tabular}


Mismatched Filter Design using Improved Cuckoo Search Algorithm for Optimum Detection

\begin{tabular}{|l|c|c|c|c|c|c|c|}
\hline 150 & -19.4394 & 0.1067 & -20.3384 & -22.2293 & -25.1466 & -25.1466 & -25.1854 \\
\hline 200 & -20 & 0.1000 & -20.9840 & -23.2085 & -23.7544 & -26.6161 & -25.8020 \\
\hline 250 & -20 & 0.1000 & -21.1397 & -22.6288 & -23.2185 & -25.9349 & -27.0647 \\
\hline 300 & -20.5993 & 0.0933 & -21.6126 & -23.5952 & -24.0201 & -25.7413 & -27.8273 \\
\hline
\end{tabular}

Table. II Improvement of PSLR of Ternary Sequence by using cascaded MMF along with adaptive filter

\begin{tabular}{|c|c|c|c|c|c|c|c|}
\hline $\begin{array}{c}\text { Sequence } \\
\text { Length }\end{array}$ & $\begin{array}{c}\text { PSLR of } \\
\text { MF (dB) }\end{array}$ & $\begin{array}{c}\text { MF } \\
\text { ASP }\end{array}$ & $\begin{array}{c}\text { PSLR of } \\
\text { DE MMF } \\
(\mathbf{d B})\end{array}$ & $\begin{array}{c}\text { PSLR of } \\
\text { Cascaded } \\
\text { MMF using } \\
\text { DE \& Basic } \\
\text { CS (dB) }\end{array}$ & $\begin{array}{c}\text { PSLR of } \\
\text { Cascaded } \\
\text { MMF using } \\
\text { DE \& New } \\
\text { CS (dB) }\end{array}$ & $\begin{array}{c}\text { PSLR of } \\
\text { Adaptive } \\
\text { Filter with } \\
\text { LMS (dB) }\end{array}$ & $\begin{array}{c}\text { PSLR of } \\
\text { Adaptive } \\
\text { Filter with } \\
\text { BSSLMS } \\
\text { (dB) }\end{array}$ \\
\hline 20 & -17.5012 & 0.1333 & -24.7471 & -27.8679 & -28.3686 & -54.7257 & -44.1132 \\
\hline 25 & -16.4782 & 0.1500 & -20.9613 & -23.7410 & -24.9498 & -27.1811 & -33.2651 \\
\hline 30 & -17.6921 & 0.1304 & -22.4835 & -25.6221 & -27.5755 & -31.3481 & -31.3134 \\
\hline 35 & -18.4164 & 0.1200 & -22.0565 & -25.8317 & -26.9208 & -31.2022 & -31.0419 \\
\hline 40 & -17.7860 & 0.1290 & -19.6416 & -22.3772 & -23.2778 & -25.7907 & -26.3466 \\
\hline 45 & -16.6502 & 0.1471 & -20.2623 & -23.7721 & -24.8530 & -26.3350 & -27.0468 \\
\hline 50 & -18.8402 & 0.1143 & -21.4103 & -25.3260 & -26.1769 & -28.5849 & -28.6465 \\
\hline 60 & -17.6921 & 0.1304 & -19.2557 & -21.6970 & -22.9043 & -24.6368 & -25.1839 \\
\hline 70 & -18.5884 & 0.1176 & -19.1906 & -21.6211 & -22.2890 & -24.3113 & -24.3485 \\
\hline 80 & -18.2155 & 0.1228 & -19.2875 & -21.6121 & -22.1114 & -24.1106 & -25.0101 \\
\hline 90 & -18.4597 & 0.1194 & -19.6799 & -22.3450 & -23.6101 & -26.1876 & -26.4284 \\
\hline 100 & -18.5314 & 0.1184 & -19.5293 & -21.7585 & -22.6794 & -24.3237 & -24.8869 \\
\hline 150 & -19.2442 & 0.1091 & -20.0372 & -22.3036 & -22.3024 & -24.4962 & -24.9883 \\
\hline 200 & -20.2848 & 0.0968 & -21.3104 & -23.9837 & -24.0160 & -25.7698 & -26.0474 \\
\hline 250 & -19.8618 & 0.1016 & -21.0719 & -23.6067 & -23.8606 & -26.3055 & -27.3435 \\
\hline 300 & -20.5993 & 0.0933 & -21.3824 & -23.3010 & -24.2622 & -24.7402 & -25.9931 \\
\hline
\end{tabular}

For sequence length 13, PSLR obtained for binary and ternary code in matched filter response is -22.2789 and -20 $\mathrm{dB}$. This value is optimized to $-40.2502 \mathrm{~dB}$ and $-33.7014 \mathrm{~dB}$ with basic cuckoo search algorithm. However, with modified $\mathrm{CS}$ it is reduced further to $-41.2058 \mathrm{~dB}$ and $-34.2495 \mathrm{~dB}$. The comparison of binary and ternary codes for different length is shown in Fig.2 and Fig.3.

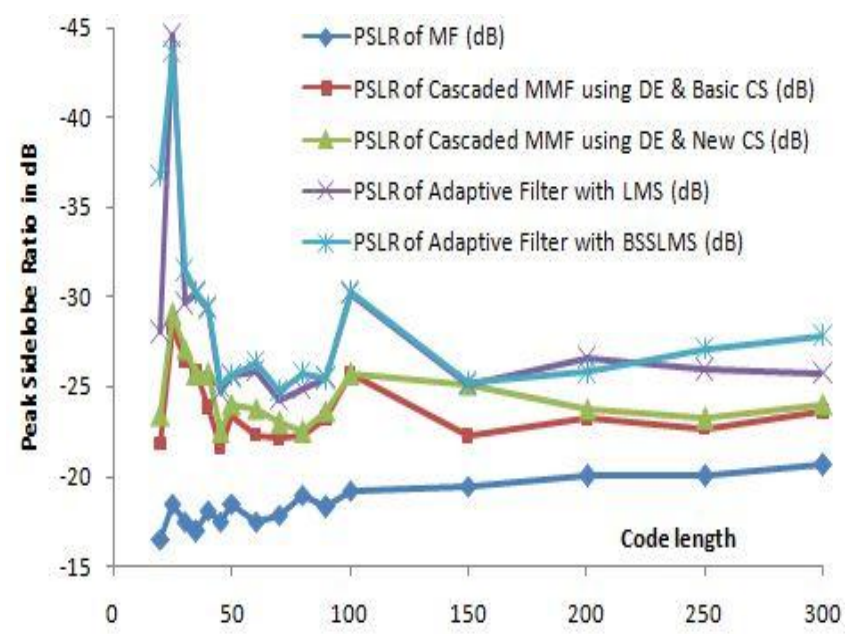

Fig.2 Performance of Binary code

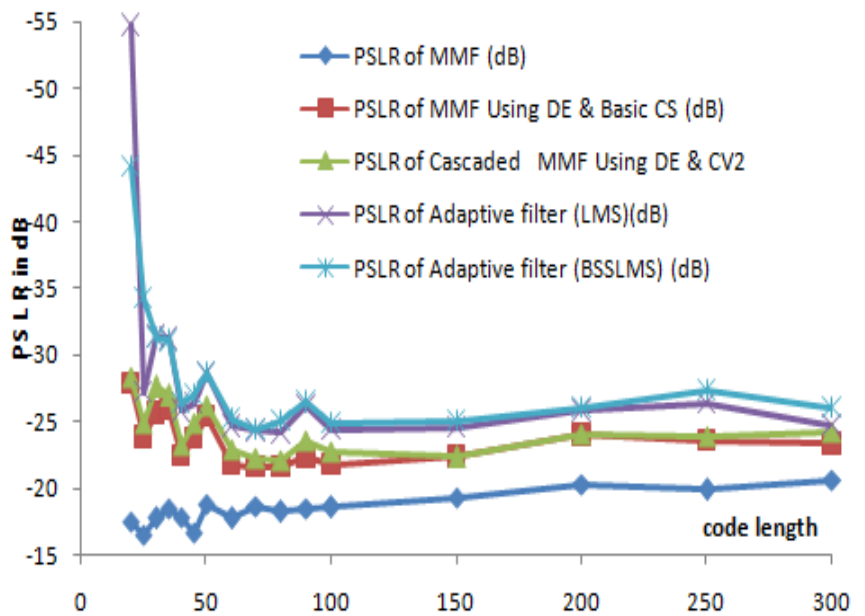

Fig.3 Performance of Ternary Sequence

The results obtained at the output of each filter for sequence length 300 is shown in Fig.4 

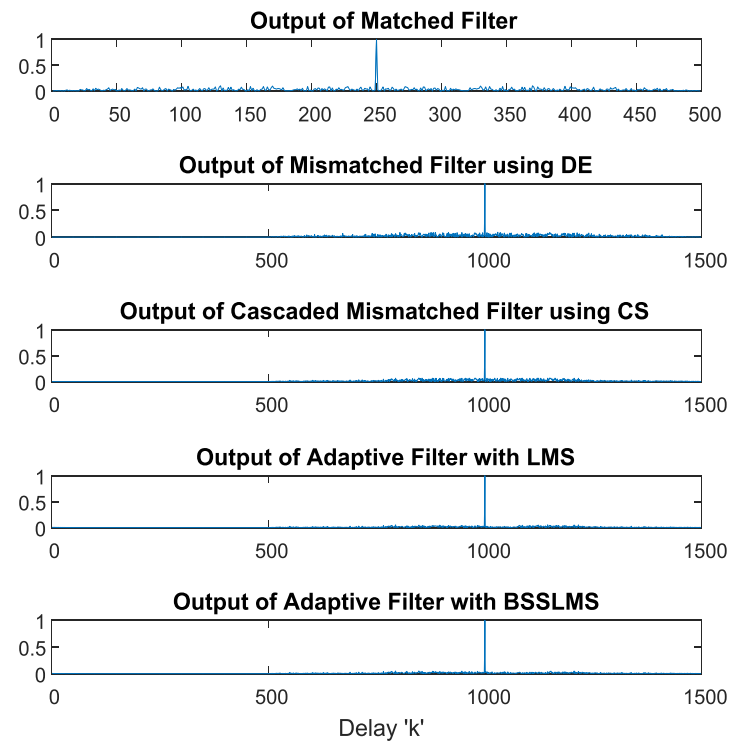

Fig.4 Output response of MF, MMF and adaptive filter

\section{CONCLUSION}

This paper deals with enhancement of performance of binary and ternary sequences with modified cuckoo search algorithm. The efficiency of cuckoo search lies in local and global search which depends on probability distribution function such as Gauss, Cauchy and Gamma function that helps in determining random walk step sizes. To avoid the problem of premature convergence, Cauchy operator is used instead of Levy distribution in spite of its better exploring capability in the search space. The modified version of cuckoo search employs grouping strategy which helps in improving the exploration and exploitation tendency. The grouping makes the confined search using different equations for each group of population and increase the performance. From the simulation results it is observed that the peak sidelobe ratio of binary and ternary code for length 50 is reduced to -23.9746 and -26.1769 with the proposed technique. These values are further improved to -25.5984 and -28.6465 by the use of adaptive filters after mismatched filter.

This algorithm is most efficient compared to PSO and DE which is reported in literature survey. The contribution of this paper is to design mismatched filter coefficients which provides good improvement in peak sidelobe ratio for lower length sequences. Because larger length sequence requires proportionally larger length mismatched filter which increases the design complexity. This work can be extended by modifying the value of switching parameter linearly, exponentially and with increasing powers.

\section{REFERENCES}

[1] M I Skonik, "Introduction to Radar Systems" Tata McGraw Hill Book Co., New York, 3rd Ed., 2001.

[2] E Mozeson, N Levanon, Radar Signals, Hoboken, NJ: John Wiley \& Sons, Inc. 2004.

[3] A H Rahman, H Elbardawiny, A Sobhy, F M Ahmed and M Hassan, "A Novel sidelobe cancellation method for binary barker code pulse compression," Proceeding of 17 th International conference on Aerospace sciences and aviation technology, ASAT-17, 11-13 April 2017, pp. 1-10.

[4] B kiranmai and P Rajesh kumar, "Performance evaluation of compound barker codes using cascaded mismatched filter technique," International Journal of computer applications, vol.121-no.19, July 2015, pp.31-34.
[5] Rohling, H., and Plagge, W., "Mismatched-Filter Design for Periodic Binary Phased Signals", IEEE Transactions on Aerosp Electron. Systems, vol. AES-25, No.6, November 1989, pp. 890-897.

[6] C Nunn, "Constrained optimization applied to pulse compression codes and filters,” IEEE Int. Radar Conf., 9-12 May 2005, pp. 190-194.

[7] N Levanon, "Cross Correlation of long binary signals with long mismatched filters,” IEE Proc.-Radar and Sonar navig., July 2005, pp.1-6.

[8] S Saremi, S Mirjalili and A Lewis, "Biogeography-based optimisation with chaos," Neural Computing \& Applications, vol.25, no. 5, 2014, pp. 1077-1097.

[9] Z Y Guo, B Chen, M. Ye, and B. Cao, "Self-adaptive chaos differential evolution," in Advances in Natural Computation: Second International Conference, ICNC 2006, China, September 24-28, 2006. Proceedings, Part I, L. Jiao, L. Wang, X.-B. Gao, J. Liu, and F. Wu, Eds., vol. 4221 of Lecture Notes in Computer Science, Springer, Berlin, Germany, 2006, pp. 972-975.

[10] R Ebrahim zadeh and M Jampour, "Chaotic genetic algorithm based on Lorenz chaotic system for optimization problems," International Journal of Intelligent Systems and Applications, vol. 5, no. 5, 2013, pp. 19-24.

[11] A H Gandomi, X S Yang, S Talatahari and A H Alavi, "Firefly algorithm with chaos," Communications in Nonlinear Science and Numerical Simulation, vol. 18, no. 1, pp. 89-98, 2013.

[12] K. Renu and P. Rajesh Kumar, "Performance of Ternary Sequences using Adaptive Filter," ICMEET-2017, Proceedings of Springer conference LNEE series 471, 8-9 September 2017, pp.63-74.

[13] K. Renu and P. Rajesh Kumar, "Improvement in performance of Ternary Sequence using Binary Step Size LMS Algorithm," ICMEET-2018, Proceedings of Springer conference LNEE series 521. pp. 161-170.

[14] S Das, A Abraham and A Konar, "Particle Swarm Optimization and Differential Evolution Algorithms: Technical Analysis, Applications and Hybridization Perspectives," Studies in Computational Intelligence (SCI) 116, 2008, pp.1-38.

[15] P Srihari and K Raja Rajeswari, "Development and performance evaluation of PSO based adaptive mismatch filter for radar pulse compression," Proceedings of 9th international radar symposium India-2013, 10-14 December 2013, pp.1-4.

[16] X S Yang and S Deb, "Cuckoo search via Lévy flights," in Proceedings of the World Congress on Nature \& Biologically Inspired Computing (NABIC '09), IEEE, Coimbatore, India, December 2009, pp. 210-214.

[17] X S Yang and S Deb, "Engineering optimisation by cuckoo search," International Journal of Mathematical Modelling and Numerical Optimisation," vol. 1, no. 4, 2010, pp. 330-343.

[18] A H Gandomi, X S Yang and A H Alavi, "Cuckoo search algorithm: a metaheuristic approach to solve structural optimization problems," Engineering with Computers, vol. 29, no. 1, 2013, pp. 17-35.

[19] L Huang, S Ding, S Yu, J Wang and K Lu, "Chaos-enhanced cuckoo search optimization algorithms for global optimization," Applied Mathematical Modelling, 2016, 40 (5), 3860-3875.

[20] R Salgotra, U Singh, S Saha, "New Cuckoo Search Algorithms with enhanced exploration and exploitation properties", Experts systems with Applications 95, Nov, 2017, pp. 384-420.

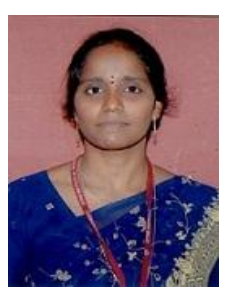

\section{AUTHORS PROFILE}

K. Renu, she did her M. Tech from Andhra University Visakhapatnam. At present She is pursuing her $\mathrm{Ph} . \mathrm{D}$ from the same. She is working as an Assistant Professor in ECE Department, GIT, GITAM (Deemed to be University), Visakhapatnam, A.P, India. Her research interests Radar Signal Processing techniques and its application to sonar and spread spectrum communication. She has 12 years of experience in Teaching for UG and PG level. She has life membership in Institute of Engineers and international Association of Engineers.

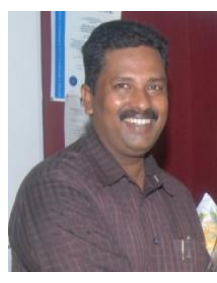

Prof. P. Rajesh Kumar honoured with Ph.D from AUCE, Andhra University, Visakhapatnam, A.P, India in 2006. He published many research papers in different international/national journals and also conferences. Presently he is a professor in the Dept. of ECE, AUCE, Andhra University, Visakhapatnam, India. He guided 10 Ph.D theses. He is having 23 years of experience in teaching and 14 years in research. His research field of interest includes Signal processing in Radar, Communication theory and its applications, Antennas, Image processing. He has been the Chairman of BOS in dept. of ECE, A.U since 2017. 